\section{Gyroscope precession in special and general relativity from basic princi- ples}

\author{
Rickard M. Jonsson
}

Department of Theoretical Physics, Physics and Engineering Physics, Chalmers University of Technology, and Göteborg University, 41296 Gothenburg, Sweden

E-mail: rico@fy.chalmers.se

Submitted: 2004-12-09, Published: 2007-05-01

Journal Reference: Am. Journ. Phys. 75463

\begin{abstract}
In special relativity a gyroscope that is suspended in a torque-free manner will precess as it is moved along a curved path relative to an inertial frame $S$. We explain this effect, which is known as Thomas precession, by considering a real grid that moves along with the gyroscope, and that by definition is not rotating as observed from its own momentary inertial rest frame. From the basic properties of the Lorentz transformation we deduce how the form and rotation of the grid (and hence the gyroscope) will evolve relative to $S$. As an intermediate step we consider how the grid would appear if it were not length contracted along the direction of motion. We show that the uncontracted grid obeys a simple law of rotation. This law simplifies the analysis of spin precession compared to more traditional approaches based on Fermi transport. We also consider gyroscope precession relative to an accelerated reference frame and show that there are extra precession effects that can be explained in a way analogous to the Thomas precession. Although fully relativistically correct, the entire analysis is carried out using three-vectors. By using the equivalence principle the formalism can also be applied to static spacetimes in general relativity. As an example, we calculate the precession of a gyroscope orbiting a static black hole.
\end{abstract}

\section{Introduction}

In Newtonian mechanics a spinning gyroscope, suspended such that there are no torques acting on it, keeps its direction fixed relative to an inertial system as we move the gyroscope around a circle. However, in special relativity the gyroscope will precess, meaning that the direction of the gyroscope central axis will rotate, see Fig. 1 .

If we denote the circle radius by $R$ and the gyroscope velocity by $v$ we can express the angular velocity $\Omega$ of the gyroscope precession as $\sqrt{1}$

$$
\Omega=(\gamma-1) \frac{v}{R}
$$

Here $\gamma=1 / \sqrt{1-v^{2} / c^{2}}$ and $c$ is the speed of light. Henceforth, unless otherwise stated, we will for convenience set $c=1$. The precession given by Eq. (11) is known as Thomas precession』2 In particular we note that for $v \ll 1$ and $\gamma \simeq 1$, the right-hand side of Eq. (11) tends to be very
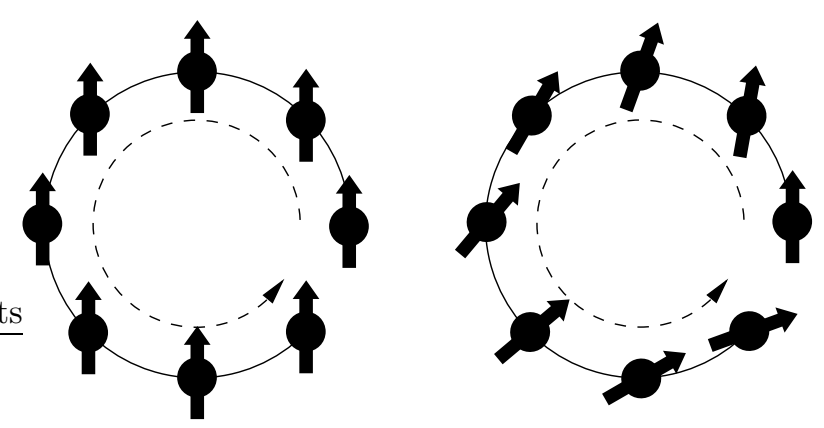

Figure 1: A gyroscope transported around a circle. The vectors correspond to the central axis of the gyroscope at different times. The Newtonian version is on the left, the special relativistic version is on the right.

small. Thus to obtain a substantial angular velocity due to this relativistic precession, we must have very high velocities (or a very small circular radius).

In general relativity the situation becomes even more interesting. For instance, we may consider a gyroscope orbiting a static black hole at the photon radius (where free photons can move in circles) $\sqrt[3]{3}$ The gyroscope will precess as depicted in Fig.2 2 independently of the velocity.

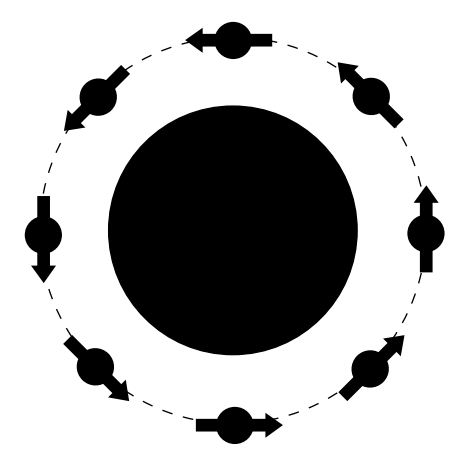

Figure 2: A gyroscope transported along a circle at the photon radius of a static black hole. The gyroscope turns so that it always points along the direction of motion.

How a gyroscope precesses for these examples can be derived using four-vectors and Fermi transport [4 Although the Fermi approach is very general, it typically results in a set of coupled differential equations that are rather complicated and do not provide much physical insight (see Appendix A).

In the following we will take a different approach. We start by discussing why there is Thomas precession in special relativity. We also derive the exact relation, Eq. (1), using only rudimentary knowledge of special relativity. We then consider gyroscope precession with respect to an accelerated reference frame within special relativity. We show that if the gyroscope moves inertially, but the reference frame accelerates perpendicularly to the gyroscope direction of motion, the gyroscope will precess relative to the reference frame.

As an application where both the reference frame and 
the gyroscope accelerates we will consider a gyroscope on a train that moves along an upward accelerating platform as shown in Fig. 3 .

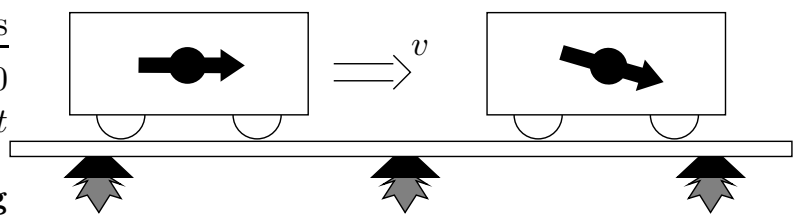

Figure 3: A train at two consecutive times moving with velocity $v$ relative to a platform that accelerates upward. A gyroscope with a torque free suspension on the train will precess clockwise for $v>0$.

If we neglect the Earth's rotation, an ordinary platform on Earth behaves just like an accelerated platform in special relativity (the equivalence principle),5 so the result can be applied to every day scenarios. The equivalence principle also allows us to apply the analysis to a gyroscope orbiting a black hole.

Sections IVI assume knowledge of special relativity. Knowledge of general relativity is assumed in Secs. VIIIX.

\section{The gyroscope grid}

Although spinning gyroscopes are the typical objects of interest when discussing relativistic precession effects, we will in the following consider a grid (say of metal) that we call the gyroscope grid. This grid is by definition not rotating as observed from its own momentary inertial rest frame. The central axis of an ideal gyroscope with a torque-free suspension is, by definition, also non-rotating as observed from its own momentary inertial rest frame. It follows that the axis of an ideal gyroscope, which is transported together with the grid, will keep its direction fixed relative to the grid. Thus, the precession of an actual gyroscope (assuming it behaves like an ideal gyroscope) follows from the behavior of the gyroscope grid. The use of the grid will also allow us to put the effects of precession due to the gyroscope grid acceleration on an equal footing with the precession effects that come from the acceleration of the reference frame.

\section{A The boost concept}

In special relativity, a Lorentz transformation to a new set of coordinates, which are non-rotated relative to the original coordinates, is known as a boost of the coordinates 6 Equivalently a physical boost of an object can be performed. As seen by an observer at rest in a certain inertial reference frame, a physical boost of an object by a certain velocity is equivalent to performing a boost of the observer's reference frame by minus the velocity (while not physically affecting the object). In particular, boosting an object with respect to the object's initial rest frame means giving the object the velocity of the boost and length contracting the object along the direction of motion. At times we will use the term pure boost to stress the non-rotating aspect of the boost. We will also assume that any real push of an object (such as the gyroscope grid), works like a pure boost relative to the momentary inertial rest frame of the object.

We will next illustrate how three consecutive physical boosts of a grid, where the boosts are each non-rotating as observed from the momentary rest frame of the grid, will result in a net rotation. The result can be formally derived by making successive Lorentz transformations (multiplying matrices), but from the derivation in the next section we can also understand how the rotation arises. The net resulting rotation is the key to the Thomas precession as presented here.

\section{B The effect of three boosts}

Consider a grid in two dimensions, initially at rest and non-rotating relative to an inertial system $S$. We then perform a series of boosts of the grid as sketched in Fig. 4 . Note especially what happens to the thick bar and its end points.

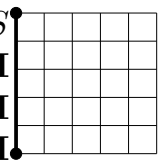

(a)

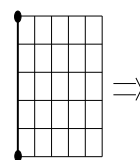

(b)

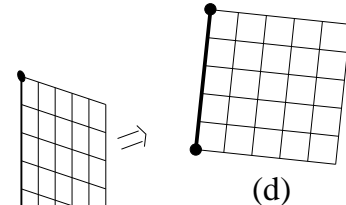

(c)
Figure 4: (a) The grid at rest with respect to $S$. (b) The grid after a pure boost with velocity $v$ to the right, relative to $S$. Note the length contraction. (c) The grid after a pure upward boost relative to a system $S^{\prime}$ that moves with velocity $v$ to the right. (d) The grid after a pure boost that stops the grid relative to $S$.

After the first boost by a velocity $v$ to the right, the grid is at rest relative to another inertial system $S^{\prime}$. The grid is then given a pure upward boost relative to $S^{\prime}$ by a velocity $\delta u^{\prime}$. Relative to the original system $S$, the grid will then move in a direction $\hat{\mathbf{n}}$ up and to the right. Through the upward boost the originally vertical grid bars remain vertical relative to $S^{\prime}$; thus they will also remain vertical relative to $S$, as follows from the Lorentz transformation. However, the originally horizontal bars will become rotated. To understand this rotation, consider all of the events along a horizontal bar just as the bar starts moving upward relative to $S^{\prime}$. These events are all simultaneous relative to $S^{\prime}$, but relative to $S$ the rearmost event (the leftmost event) will happen first (relativity of simultaneity). Thus the leftmost part of the bar 
will have a head start (upward) relative to the rightmost part, and the bar will therefore become rotated. Finally, we stop the grid, in other words we make a pure boost in the $-\hat{\mathbf{n}}$ direction, so that the grid stops relative to $S$. The effect will be to remove the length contraction in the $\hat{\mathbf{n}}$ direction, that is, to stretch the grid in the $\hat{\mathbf{n}}$ direction. Through this stretching we understand that the originally vertical grid bars will rotate clockwise. Because none of the boosts deform the grid as observed in the grid's own momentary rest frame, it follows that the entire final grid will be rotated clockwise relative to the original grid.

\section{Calculating the precise turning angle}

The upward boost by a velocity $\delta u^{\prime}$ relative to $S^{\prime}$ yields an upward velocity $\delta u=\delta u^{\prime} / \gamma$ (time dilation) as observed from $S$. Consider now two points separated by a distance $L_{0}$ along an originally vertical bar of the grid, as measured in the grid's own frame. As observed in $S^{\prime}$, the distance between the points after the upward boost is, due to length contraction, given by $L=L_{0} / \gamma\left(\delta u^{\prime}\right)$. This distance is also the distance between the points as observed in $S$, as follows from the Lorentz transformation. Also, the velocity of the points after the upward boost is $v \hat{\mathbf{x}}+\delta u \hat{\mathbf{y}}$ as observed in $S$.

When we stop the grid, the length expansion (that is, the removal of length contraction) will shift the topmost point relative to the lowest point, resulting in a rotation by an angle $\delta \alpha$ as depicted in Fig. 5. From the definitions in Fig. 5 it follows that

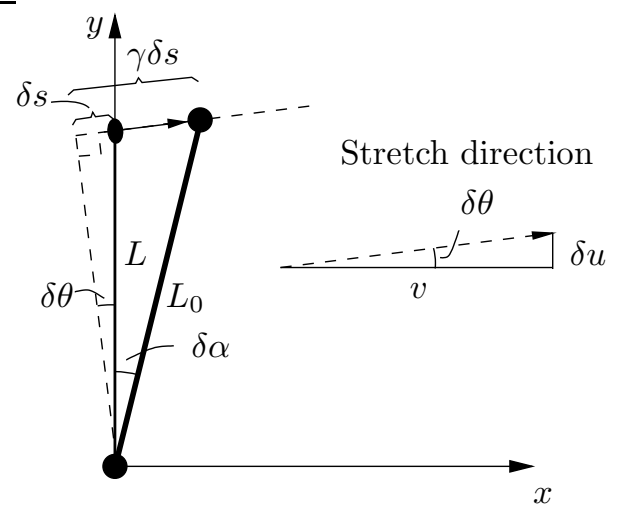

Figure 5: The stretch-induced tilt of the two points (the filled circles) due to the final stopping of the grid. The distance between the points prior to the stretching, as measured in the direction of motion, is denoted by $\delta s$.

$$
\tan \delta \theta=\frac{\delta u}{v}, \quad \sin (\delta \alpha+\delta \theta)=\frac{\gamma \delta s}{L_{0}}, \quad \sin \delta \theta=\frac{\delta s}{L} .
$$

From now on we will assume that $\delta u$ is infinitesimal. Because the $\gamma$-factor entering the relation between $L$ and $L_{0}$ depends on $(\delta u)^{2}$, we have $L=L_{0}$ to first order in $\delta u$. It follows that, to first order in $\delta u$, Eq. (2) reduces to

$$
\delta \theta=\frac{\delta u}{v}, \quad \delta \alpha+\delta \theta=\frac{\gamma \delta s}{L_{0}}, \quad \delta \theta=\frac{\delta s}{L_{0}} .
$$

From Eq. (3) we find

$$
\delta \alpha=\frac{\delta u}{v}(\gamma-1)
$$

Here $\delta \alpha$ is the resultant clockwise angle of rotation of the grid, after the three consecutive boosts. The result also applies to a grid that was initially rotated by a certain angle relative to the grid we considered above. To see this, suppose that we perform the three boosts simultaneously on the two grids. Because the boosts are all non-rotating as observed in the momentary rest frame of the grids, the relative angle between the grids must be preserved. Thus Eq. (41) gives the angle of rotation resulting from the three boosts in question, regardless of the initial rotation of the grid.

For an infinitesimal boost in a general direction relative to $S^{\prime}$, only the upward directed part of the boost contributes to the rotation 7 Thus Eq. (4) holds also for this case if we interpret $\delta u$ in Eq. (4) as the part of the infinitesimal velocity change that is perpendicular to the direction of motion.

\section{The uncontracted grid}

For a grid in motion relative to a certain specified reference frame (for example an inertial frame), we now introduce what we call the uncontracted grid. This grid is obtained by imagining the real grid without length contraction along the direction of motion. The idea is illustrated in Fig. 6.
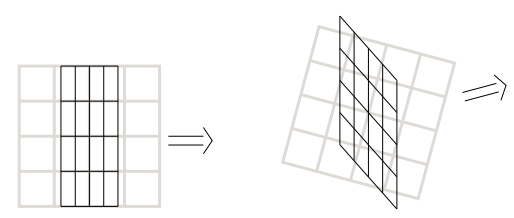

Figure 6: The real grid (black thin lines) and the corresponding imagined uncontracted grid (grey thick lines) before and after a large upward boost.

From Fig. 4, we note that the imagined uncontracted grid, immediately before and after the infinitesimal upward boost, is identical (in form and rotation) to the initial and the final actual grid respectively. From the discussion at the end of Sec. II C it therefore follows that for any real grid moving on a plane that receives an infinitesimal boost (non-rotating as observed in the grid's own momentary inertial rest frame) by a velocity $\delta u$ perpendicular to the direction of motion, the corresponding uncontracted grid will rotate an angle given by Eq. (4) as

$$
\delta \alpha=\frac{\delta u}{v}(\gamma-1)
$$


Henceforth we will always describe the gyroscope grid in terms of the uncontracted grid. If we have found the evolution of the uncontracted grid for a particular path, we can always find the observed real grid by length contracting the uncontracted grid in the momentary direction of motion.

\section{E Circular motion}

Consider a gyroscope grid moving with velocity $v$ along a circle of radius $R$. During a time step $\delta t$, the grid receives an infinitesimal boost perpendicular to the direction of motion. In the inertial frame of the circle the perpendicular velocity change is given by

$$
\delta u=\frac{v^{2}}{R} \delta t
$$

The corresponding uncontracted grid will rotate an angle according to Eq. (5) during the boost. At the next time step there is a new boost and a new induced rotation. It follows that there is an ongoing precession of the uncontracted grid as depicted in Fig. 7. The angular velocity of rotation is given by Eqs. (5) and (6) as

$$
\frac{\delta \alpha}{\delta t}=(\gamma-1) \frac{v}{R}
$$

Thus we have derived the Thomas precession given by Eq. (1). Note that Eq. (7) describes how fast the imagined uncontracted grid rotates.

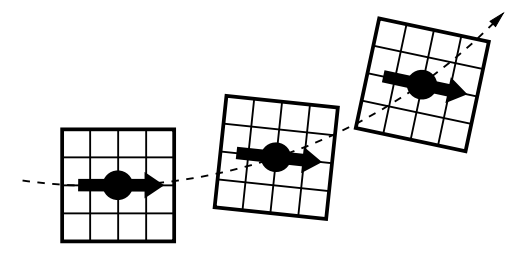

Figure 7: A gyroscope grid at successive time steps. Both the grid and the gyroscope are depicted as they would be observed if they were uncontracted.

\section{F The mathematical advantage of the un- contracted grid}

We have shown that the uncontracted grid evolves according to a simple law of rotation. The central axis of a gyroscope, if it were not length contracted along the direction of motion, obeys the same simple law of rotation. The actual axis of a gyroscope, however, changes its length over time, and its angular velocity would not be as simple as that given by Eq. (7). A differential equation for the evolution of the actual axis, would hide the simple dynamics of a rotation and a superimposed length contraction, which would complicate the analysis. Similarly, the standard approach to calculating gyroscope precession, which uses the Fermi transport equation for the spin vector of the gyroscope, is also comparatively complicated (see Appendix $\mathrm{A}$ and Ref. 8 for further details).

\section{G Comments on the uncontracted grid}

Although we may think of the uncontracted grid as a mathematically convenient intermediate step in finding the actual grid, there is more to this concept. As follows from its definition, the uncontracted grid corresponds directly to the grid as experienced in a system that moves with the grid and that is related to the reference frame in question by a pure boost.

Consider a special relativistic scenario of a gyroscope grid suspended in a torque free manner inside a satellite. The satellite uses its jet engines to move along a smooth simple closed curve on a plane. We want to measure from the satellite the precession angle of the gyroscope grid after a full orbit. If we assume that there are a couple of suitably placed fixed stars, we can use their direction as observed from the satellite at the initial and final point of the orbit (which coincide), as guidelines to establish a reference system within the satellite. For this scenario the uncontracted grid is the physical object in which we are interested, because it's orientation precisely corresponds to the orientation of the actual gyroscope grid relative to the star calibrated reference frame of the satellite. In particular, if the uncontracted grid has rotated a certain angle after the full orbit, so has the actual gyroscope grid as measured from the satellite.

\section{Boosting the reference frame}

Now let us consider the effect of a boost of the reference frame rather than of the gyroscope grid. To make the analogy with the discussion in Sec. II clearer, we consider a real grid as a reference frame. We assume that the reference frame initially is at rest relative to an inertial system $S$, and is then boosted upward so that it is at rest with respect to another inertial system $S^{0}$, see Fig. 8. The gyroscope grid is assumed to move with constant speed $v$ to the right as observed in $S$.
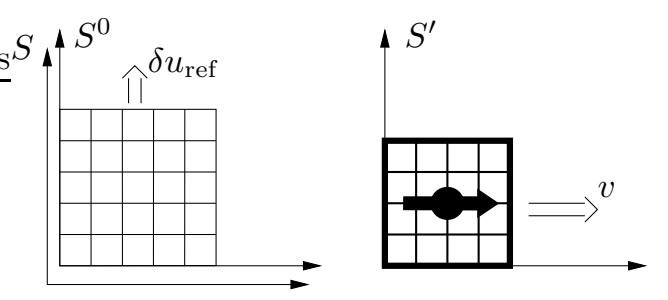

Figure 8: Boost of the reference frame (of which the depicted thin grid is a small part) upward by a velocity $\delta u_{\text {ref. }}$. The velocity of the gyroscope grid is maintained.

Relative to the gyroscope system, the reference frame initially moves to the left, and is then (due to the boost) 
given an upward velocity $\delta u_{\text {ref }} / \gamma$ (time dilation). Because the reference frame moves relative to the gyroscope system, the reference frame is length contracted along the direction of motion. However, we can imagine the reference frame without the length contraction. Analogous to the discussion in Sec. III, the uncontracted reference frame will rotate during the boost, as depicted in Fig. 9 .

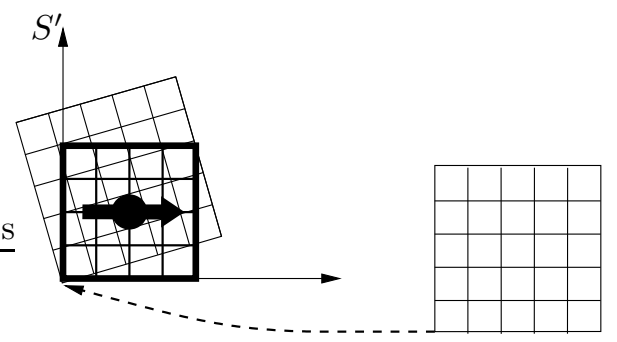

Figure 9: Relative to the gyroscope system, the reference frame (thin lines), of which we illustrate a certain part, rotates during the boost. The reference frame is depicted as it would appear if it was not length contracted relative to the gyroscope system.

The net counterclockwise angle of rotation for the reference frame is found by substituting $\delta u$ by $\delta u_{\text {ref }} / \gamma$ into Eq. (5):

$$
\delta \alpha=\frac{\delta u_{\mathrm{ref}}}{\gamma} \frac{\gamma-1}{v} .
$$

Note that removing the length contraction of the uncontracted reference frame relative to the gyroscope grid yields the same relative configuration as removing the length contraction of the gyroscope grid relative to the reference frame. It follows that the upward boost of the reference frame yields a clockwise rotation of the uncontracted gyroscope grid, described by Eq. (8), relative to the reference frame. This relative rotation is precisely the rotation in which we are interested. Note in particular that an upward boost of the reference frame yields a clockwise relative rotation just as an upward boost of the gyroscope grid yields a clockwise relative rotation.

\section{Three dimensions}

In the two-dimensional reasoning of Secs. II and III, the induced rotation occurred in a plane spanned by the velocity vector and the vector for the velocity change. For more general three-dimensional motion and velocity changes, the induced rotation should still occur in a plane spanned by these two vectors. The axis of rotation can therefore be expressed in terms of the cross product of these two vectors. Let us introduce $\delta \boldsymbol{\alpha}$ as a vector whose direction indicates the axis of rotation and whose magnitude corresponds to the angle of rotation for the uncontracted gyroscope grid relative to the reference frame. Also, let $\mathbf{v}$ be the velocity vector of the gyroscope relative to the reference frame. The three-vector analog of Eq. (5) for a velocity change $\delta \mathbf{u}_{\text {gyro }}$ of the gyroscope, using the identity $(\gamma-1)=\gamma^{2} v^{2} /(\gamma+1)$, can be written as

$$
\delta \boldsymbol{\alpha}=\frac{\gamma^{2}}{\gamma+1}\left(\delta \mathbf{u}_{\mathrm{gyro}} \times \mathbf{v}\right) .
$$

The corresponding vector analog of Eq. (8) for a velocity change $\delta \mathbf{u}_{\text {ref }}$ of the reference frame is given by

$$
\delta \boldsymbol{\alpha}=\frac{\gamma}{\gamma+1}\left(\delta \mathbf{u}_{\mathrm{ref}} \times \mathbf{v}\right) .
$$

Note that the cross product selects only the part of the velocity change that is perpendicular to the relative direction of motion.

Consider now an infinitesimal boost of both the gyroscope and of the reference frame. If we assume that we start by boosting the gyroscope, which gives a velocity change $\delta \mathbf{u}_{\text {gyro }}$, this boost yields a rotation according to Eq. (9). Subsequently boosting the reference frame by a velocity $\delta \mathbf{u}_{\text {ref }}$ yields another rotation given by Eq. (10), but $\mathbf{v}$ should be replaced by $\mathbf{v}+\delta \mathbf{u}_{\text {gyro }}$. However, to first order in $\delta \mathbf{u}_{\text {ref }}$ and $\delta \mathbf{u}_{\text {gyro }}$ this replacement does not affect Eq. (10). Because infinitesimal rotations can be added (to first order in the magnitude of the rotations), it then follows that the net rotation is given by Eqs. (9) and (10) as

$$
\delta \boldsymbol{\alpha}=\frac{\gamma^{2}}{\gamma+1}\left(\delta \mathbf{u}_{\mathrm{gyro}} \times \mathbf{v}\right)+\frac{\gamma}{\gamma+1}\left(\delta \mathbf{u}_{\mathrm{ref}} \times \mathbf{v}\right) .
$$

Now consider a continuously accelerating reference frame and gyroscope grid. Relative to an inertial system in which the reference frame is momentarily at rest, we have $\delta \mathbf{u}_{\text {gyro }}=\mathbf{a}_{\text {gyro }} \delta t$ and $\delta \mathbf{u}_{\text {ref }}=\mathbf{a}_{\text {ref }} \delta t$ for a time step $\delta t$. We substitute these relations into Eq. (11) and obtain the net angular velocity vector $\boldsymbol{\Omega}=\delta \boldsymbol{\alpha} / \delta t$ for the gyroscope grid rotation relative to the reference frame as

$$
\boldsymbol{\Omega}=\frac{\gamma}{\gamma+1}\left[\gamma \mathbf{a}_{\mathrm{gyro}}+\mathbf{a}_{\mathrm{ref}}\right] \times \mathbf{v}
$$

Because we are interested in how the gyroscope grid rotates relative to the accelerating reference frame, it can be useful to express the motion relative to the reference frame. Consider a path with local curvature radius $R$ and curvature direction $\hat{\mathbf{n}}$, fixed to the reference frame. In Appendix $\mathrm{B}$ we show that for motion along this path we have (just like in Newtonian mechanics)

$$
\left[\mathbf{a}_{\text {gyro }}\right]_{\perp}=\left[\mathbf{a}_{\text {ref }}\right]_{\perp}+v^{2} \frac{\hat{\mathbf{n}}}{R} .
$$

Here $\mathbf{a}_{\text {gyro }}$ and $\mathbf{a}_{\text {ref }}$ refer to the accelerations relative to an inertial frame in which the reference frame is momentarily at rest. The notation $\perp$ denotes the part of the acceleration that is perpendicular to the direction of motion for the gyroscope grid. Because of the cross product in Eq. (12), the perpendicular part of the acceleration is the only part that matters for $\boldsymbol{\Omega}$. If we substitute $\mathbf{a}_{\text {gyro }}$ 
from Eq. (13) into Eq. (12) and simplify the resultant expression, we find

$$
\boldsymbol{\Omega}=(\gamma-1)\left(\frac{\hat{\mathbf{n}}}{R} \times \mathbf{v}\right)+\gamma\left(\mathbf{a}_{\mathrm{ref}} \times \mathbf{v}\right) .
$$

The first term on the right-hand side of Eq. (14) has the same form as the standard Thomas precession term given by Eq. (11). The second term corresponds to both direct effects of rotation from the reference frame acceleration and to the indirect effects of this acceleration, because the acceleration of the gyroscope grid relative to an inertial frame depends on the reference frame acceleration in this formulation. Equation (14) matches the formally derived Eq. (51) of Ref. 8 ,

\section{Applications}

In this section we discuss applications of the derived formalism, Eq. (14), for gyroscope precession relative to an accelerating reference frame.

\section{A Motion along a horizontal line}

Consider a special relativistic scenario of a train moving along a horizontal line relative to a platform that continually accelerates upward relative to an inertial frame (see Fig. 10).

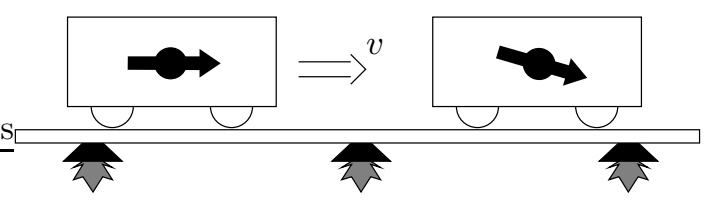

Figure 10: A train with a gyroscope moving relative to an accelerating platform observed at two successive times.

On the train a gyroscope is suspended so that there are no torques acting on it as observed from the train. For the special case of motion along a straight line relative to the reference frame (the platform in this case), we have $1 / R=0$. If we define $\mathbf{g}=-\mathbf{a}_{\text {ref }}$ as the local acceleration of an object dropped relative to the platform, Eq. (14) reduces to

$$
\boldsymbol{\Omega}=\gamma(\mathbf{v} \times \mathbf{g}) .
$$

Note that both the gyroscope and the platform reference frame accelerate with respect to an inertial frame, and hence we expect two precession effects. Both of these effects are included in the single term on the right-hand side of Eq. (15). Let $\Omega$ denote the clockwise precession rate, $v$ the velocity to the right, and $g$ the the downward acceleration of dropped object relative to the platform. Then Eq. (15) gives

$$
\Omega=\gamma v g .
$$

Note that the uncontracted grid, whose rotation with respect to the platform reference frame is given by Eq. (16), corresponds to the grid as experienced by an observer on the train (as discussed in Sec. II G). Thus we obtain the angular velocity relative to the train by multiplying the right-hand side of Eq. (16) by $\gamma$ to account for time dilation 9 Relative to the train the gyroscope thus precesses at a steady rate $\Omega_{0}$ (clockwise as depicted) given by

$$
\Omega_{0}=\gamma^{2} v g .
$$

If we assume the train velocity to be low and introduce the proper factor of $c^{2}$ to enable us to express $g$ and $v$ in SI units, we have $\Omega_{0} \approx v \mathrm{~g} / \mathrm{c}^{2}$. For a train with a velocity of $50 \mathrm{~m} / \mathrm{s}$ and a platform acceleration corresponding to that of a dropped apple on the Earth, we obtain

$$
\Omega_{0} \approx \frac{50 \frac{\mathrm{m}}{\mathrm{s}} \times 9.81 \frac{\mathrm{m}}{\mathrm{s}^{2}}}{\left(3 \times 10^{8} \frac{\mathrm{m}}{\mathrm{s}}\right)^{2}} \approx 5 \times 10^{-15} \mathrm{rad} / \mathrm{s} .
$$

This special relativistic scenario mimics a train moving along a straight platform on the Earth (neglecting the Earth's rotation). It follows that precession effects due to gravity are small for everyday scenarios on the Earth.

Because a torque-free gyroscope precesses relative to the train, it follows that the train has a proper rotation, meaning that the train rotates as observed from its own momentary inertial rest frame. This rotation can be understood without reference to the gyroscope precession. The heart of the matter lies (as is often the case) in simultaneity. Let $S$ be an inertial system where the rail of the continuously accelerating platform is at rest momentarily (at $t=0)$. As observed in $S$, the horizontal straight rail will first move downward (when $t<0$ ), decelerate to be at rest at $t=0$, and then accelerate upward. Consider now all the events along a section of the rail, when the rail is at rest in $S$. Relative to the train's momentary inertial rest frame $S^{\prime}$, which moves with velocity $v$ to the right as observed from $S$, the rightmost of the events along the rail will occur first. Thus relative to $S^{\prime}$, when the rail at the rear end of the train has no vertical motion, the rail at the front end (and thus the train's front end) will already have an upward velocity. Hence a train moving as depicted in Fig. 10 has a proper counterclockwise rotation. By this reasoning we can verify the validity of Eq. (17). We also understand that as observed from $S^{\prime}$, the rail is not straight but is curved as depicted in Fig. 111,

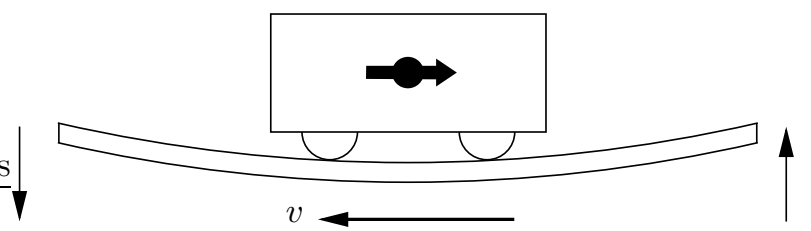

Figure 11: A sketch of the rail and the train observed from an inertial system where the train is momentarily at rest. 


\section{B Following the geodesic photon}

As another application we now study the precession of a gyroscope that follows the spatial trajectory of a free photon 10 From Eq. (B2) it follows that the trajectory of a free photon (set $v=1$ and $\mathbf{a}_{\text {particle }}=0$ ) as observed relative to an accelerating reference frame satisfies

$$
\frac{\hat{\mathbf{n}}}{R}=\mathbf{g}_{\perp} \text {. }
$$

Here $\mathbf{g}_{\perp}$ is the part of $\mathbf{g}$ that is perpendicular to $\mathbf{v}$ (recall that $\mathbf{g}=-\mathbf{a}_{\text {ref }}$ ). If we substitute the curvature given by Eq. (19) into Eq. (14), we find that the gyroscope grid angular velocity is given by

$$
\boldsymbol{\Omega}=\mathbf{v} \times \mathbf{g}_{\perp} .
$$

Consider now a normalized vector $\hat{\mathbf{t}}$ directed along the spatial direction of motion. The time derivative of $\hat{\mathbf{t}}$ relative to the reference frame satisfies $d \hat{\mathbf{t}} / d t=v \hat{\mathbf{n}} / R$. It follows that $\hat{\mathbf{t}}$ rotates with an angular velocity $\boldsymbol{\Omega}_{\hat{\mathbf{t}}}=$ $\mathbf{v} \times \hat{\mathbf{n}} / R$. If we substitute the curvature $\hat{\mathbf{n}} / R=\mathbf{g}_{\perp}$ given by Eq. (19), into this expression for $\boldsymbol{\Omega}_{\hat{\mathrm{t}}}$, we obtain

$$
\Omega_{\hat{\mathbf{t}}}=\mathbf{v} \times \mathbf{g}_{\perp} .
$$

If we compare Eqs. (21) and (20), we see that $\hat{\mathbf{t}}$ rotates with the same angular velocity as the gyroscope grid. It follows that a gyroscope transported along a spatial trajectory of a free photon will keep pointing along the direction of motion if it did initially (see Fig. 12).

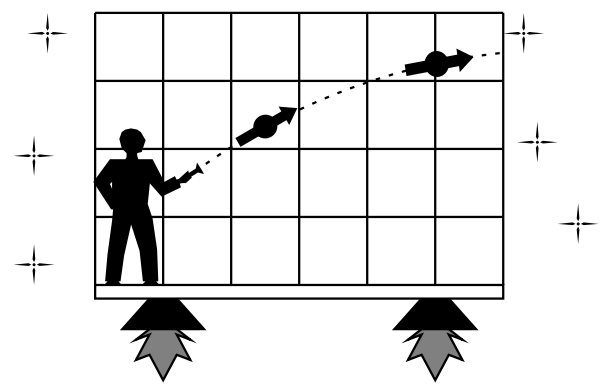

Figure 12: A free photon will in general follow a curved path relative to an accelerated reference frame. A gyroscope transported along such a path will keep pointing along the path if it did so initially.

If we imagine a static reference frame outside the event horizon of a static black hole, then locally this reference frame behaves just like an accelerated reference frame in special relativity (the equivalence principle). Hence a gyroscope outside of a black hole that follows the path of a free photon, such as a circle at the photon radius, will not precess relative to the forward direction of motion, as depicted in Fig. 2 in the introduction.

\section{Conclusions}

We have seen how the basic principles of special relativity can be used to derive a simple but exact three-vector formalism of spin precession with respect to an accelerating reference frame. The precession is given by Eq. (14) as

$$
\boldsymbol{\Omega}=(\gamma-1)\left(\frac{\hat{\mathbf{n}}}{R} \times \mathbf{v}\right)+\gamma\left(\mathbf{a}_{\mathrm{ref}} \times \mathbf{v}\right),
$$

where $\hat{\mathbf{n}} / R$ is the curvature of the gyroscope path relative to the accelerated reference frame. Recall that $\boldsymbol{\Omega}$ describes the rotation of a gyroscope axis as we imagine it without length contraction along the direction of motion. In the following, knowledge of general relativity is assumed.

\section{Axisymmetric spatial geome- tries and effective rotation vec- tors}

In a static spacetime such as that of a Schwarzschild black hole, the global static reference frame locally corresponds to the accelerated reference frames we have considered in special relativity. If we integrate the infinitesimal rotations from $\Omega$ given by either Eqs. (12) or (14), we can find the net rotation of a gyroscope that is transported along a given spatial path. Note, however, that $\boldsymbol{\Omega}$ describes how the gyroscope grid rotates relative to a frame that is parallel transported with respect to the local spatial geometry associated with the reference frame. Thus directly integrating the effects of rotation from $\boldsymbol{\Omega}$ gives the rotation relative to a frame that is parallel transported with respect to the global spatial geometry. In Fig. 13 we illustrate a section of the spatial geometry of an equatorial plane of a static black hole.

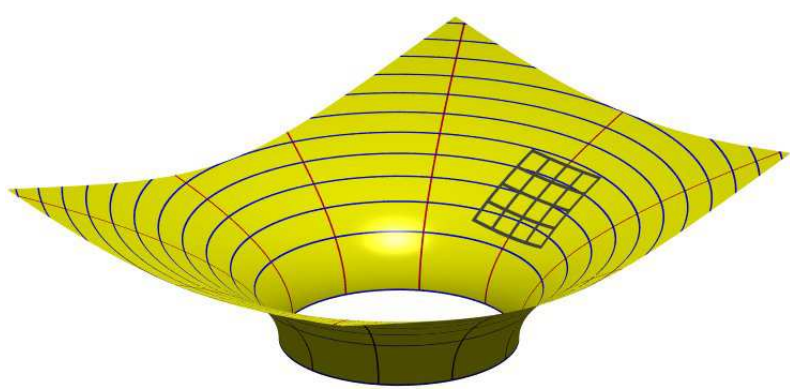

Figure 13: Sketch of the spatial geometry of a symmetry plane outside a black hole. The local static reference frame shown (the square grid) has a proper acceleration outward. For a sufficiently small such reference frame it works just like an accelerated reference frame in special relativity.

Suppose then that we consider motion in the equatorial plane of some axisymmetric geometry. For instance, 
we might be interested in the the net rotation of a gyroscope (grid) after a closed orbit around the center of symmetry (not necessarily a circular orbit). We need then take into consideration that a parallel transported frame will be rotated relative to its initial configuration after a complete orbit due to the spatial geometry. To deal with this complication we introduce a new reference frame that rotates relative to the local coordinates, spanned by the polar vectors $\hat{\mathbf{r}}$ and $\hat{\boldsymbol{\varphi}}$, in the same manner as a parallel transported reference frame does on a plane. In other words, if we consider a counterclockwise displacement $(\delta \varphi, \delta r)$, then relative to the local vectors $\hat{\mathbf{r}}$ and $\hat{\boldsymbol{\varphi}}$, the new reference frame should rotate $\delta \varphi$ clockwise. Such a "would-be-flat" reference frame always returns to its initial configuration after a full (closed) orbit.

The line element for a two-dimensional axisymmetric spatial geometry can be written in the form

$$
d s^{2}=g_{r r} d r^{2}+r^{2} d \varphi^{2} .
$$

With respect to such a geometry it is easy to show that the angular velocity of a parallel transported frame relative to a "would-be-flat" frame is given by 8

$$
\boldsymbol{\omega}_{\text {space }}=\frac{1}{r}\left(\frac{1}{\sqrt{g_{r r}}}-1\right) \mathbf{v} \times \hat{\mathbf{r}} .
$$

Because infinitesimal rotation vectors can be added (to lowest order), it follows from Eq. (24) and Eq. (14) that the gyroscope grid rotation relative to the would-be-flat frame is given by

$\boldsymbol{\Omega}_{\mathrm{eff}}=(\gamma-1)\left(\frac{\hat{\mathbf{n}}}{R} \times \mathbf{v}\right)-\gamma(\mathbf{g} \times \mathbf{v})+\frac{1}{r}\left(\frac{1}{\sqrt{g_{r r}}}-1\right) \mathbf{v} \times \hat{\mathbf{r}}$.

Alternatively we could express $\boldsymbol{\Omega}_{\mathrm{eff}}$ in terms of the gyroscope acceleration $\mathbf{a}_{\text {gyro }}$ relative to a local freely falling (inertial) frame momentarily at rest relative to the static reference frame. If we use Eq. (12) and add the rotation due to the spatial geometry as described by Eq. (24), we find

$$
\boldsymbol{\Omega}_{\mathrm{eff}}=\frac{\gamma^{2}}{\gamma+1}\left(\mathbf{a}_{\mathrm{gyro}} \times \mathbf{v}\right)-\frac{\gamma}{\gamma+1}(\mathbf{g} \times \mathbf{v})+\frac{1}{r}\left(\frac{1}{\sqrt{g_{r r}}}-1\right) \mathbf{v} \times \hat{\mathbf{r}} .
$$

Note that the time $t$ implicitly entering in Eqs. (25) and (26) through $\Omega_{\mathrm{eff}}=d \alpha / d t$ is the local proper time for a static observer. We obtain the net induced rotation of a gyroscope in closed orbit by integrating the effects of the infinitesimal rotations given by either Eq. (25) or Eq. (26).

\section{Circular orbits in static sphe- rically symmetric spacetimes}

For circular motion in a spatial symmetry plane of a static spherically symmetric spacetime, the direction of the rotation vector $\boldsymbol{\Omega}_{\mathrm{eff}}$ is constant (directed perpendicularly to the plane of motion) in the coordinate basis of the would-be-flat reference frame. For a counterclockwise motion the clockwise angular velocity of precession is then (with $\hat{\mathbf{n}}=-\hat{\mathbf{r}}$ and $\mathbf{g}=-g \hat{\mathbf{r}}$ ) given by Eq. (25) as

$$
\Omega_{\mathrm{eff}}=(\gamma-1) \frac{v}{R}-\gamma g v+\frac{v}{r}\left(\frac{1}{\sqrt{g_{r r}}}-1\right) .
$$

It is easy to show that the curvature radius of a circle at a certain $r$, for a geometry of the form of Eq. (23), is given by $R=r \sqrt{g_{r r}}$. If we substitute this result into Eq. (27), we find

$$
\Omega_{\mathrm{eff}}=\frac{v}{r}\left(\frac{\gamma}{\sqrt{g_{r r}}}-1\right)-\gamma g v .
$$

For a general spherically symmetric static spacetime, the line element of a radial line can be written in the form

$$
d \tau^{2}=g_{t t}(r) d t^{2}-g_{r r}(r) d r^{2} .
$$

Note that $g_{r r}$ is positive as defined here (to match the definition in Sec. VII $)$. From Eq. (29) it is easy to derive the local acceleration of a freely falling particle momentarily at rest. The result is

$$
g=\frac{1}{2 g_{t t} \sqrt{g_{r r}}} \frac{\partial g_{t t}}{\partial r} .
$$

So here we have an explicit expression for the $g$ which enters the expression for $\Omega_{\text {eff }}$ in Eq. (28). We are now ready to consider a specific example.

\section{A The Schwarzschild black hole}

For a Schwarzschild black hole (using standard coordinates and $c=G=1$ ) we have

$$
\begin{aligned}
g_{t t} & =(1-2 M / r) \\
g_{r r} & =(1-2 M / r)^{-1} .
\end{aligned}
$$

We substitute these two expressions into Eq. (30) and find

$$
g=\frac{M}{r^{2} \sqrt{1-2 M / r}}
$$

If we use Eq. (32) and Eq. (31b) in Eq. (28), we obtain

$$
\Omega_{\mathrm{eff}}=\frac{\gamma v}{r \sqrt{1-2 M / r}}(1-3 M / r)-\frac{v}{r} .
$$

Equation (33) gives the precession rate as a function of $r$ and $v$. For constant velocity $v$ we obtain the net rotation after a full orbit by multiplying the precession rate by the local orbital period $2 \pi r / v$. Thus we have $\alpha_{\text {per-lap }}=$ $\Omega_{\text {eff }} 2 \pi r / v$. If we use this result together with Eq. (33), we obtain for counterclockwise motion the clockwise angle of precession per lap

$$
\frac{\alpha_{\text {per-lap }}}{2 \pi}=\gamma \frac{(1-3 M / r)}{\sqrt{1-2 M / r}}-1 .
$$

In particular, for the photon radius (where geodesic photons can move on circles) at $r=3 M$, we obtain a rotation angle of $-2 \pi$ per orbit, independently of the velocity. This result is precisely what we would expect from the discussion in Sec. V B. Equation (34) is equivalent to Eq. (39) of Ref. 1112 


\section{B Geodesic circular motion}

For a free (geodesic) gyroscope in circular motion around a static black hole we have, according to Eq. (13), $v^{2} / R=$ $g$ where $R=r \sqrt{g_{r r}}$. By also using $g_{r r}$ given by Eq. (31b) and $g$ given by Eq. (32), we find the $\gamma$ factor for free circular motion:

$$
\gamma=\frac{\sqrt{1-2 M / r}}{\sqrt{1-3 M / r}} .
$$

Note that $\gamma$ becomes infinite for $r=3 M$ as it should. If we use Eq. (35) in Eq. (34), we obtain

$$
\frac{\alpha_{\text {per-lap }}}{2 \pi}=\sqrt{1-3 M / r}-1 .
$$

Equation (36) is an exact expression for the net precession angle per full orbit for an ideal gyroscope in free circular motion around a static black hole. If we assume the gyroscope to be freely "floating" within a satellite, analogous to the discussion of Sec. II G, Eq. (36) gives the rotation relative to a star-calibrated reference system of the satellite. Equation (36) matches Eq. (37) of Ref. 11.

\section{Relation to other work}

The standard approach to calculating gyroscope precession in special and general relativity is to solve the Fermi equation for the spin four-vector of the gyroscope. Even for simple applications in special relativity, such as circular motion, the resulting equations can, however, be quite complicated (see Appendix @). In general relativity, the classical approaches to gyroscope precession are based on approximations that assume "weak" gravity and small velocities (see e.g Refs. 4 and 13). The derived formalisms can therefore not be applied accurately to, for example, a gyroscope orbiting close to a black hole. Other approaches, such as that in Ref. [11, are exact but specific to circular motion. The approach of this paper, which is exact (assuming an ideal gyroscope) and applies to arbitrary motion relative to a static reference frame, is strongly linked to the more formal approaches in Refs. 8 and 14 .

\section{A The Fermi approach to circular motion}

In special and general relativity the spin of a gyroscope is represented by a four-vector $S^{\mu}$. The Fermi transport law for $S^{\mu}$ is given by

$$
\frac{D S^{\mu}}{D \tau}=u^{\mu} \frac{D u^{\alpha}}{D \tau} S_{\alpha} .
$$

Here $u^{\mu}$ is the four-velocity of the gyroscope. As a special relativistic application we consider motion with fixed speed $v$ along a circle in the $x y$-plane with an angular frequency $\omega$. We assume that the spatial part of the spin vector $\left(S^{x}, S^{y}, S^{z}\right)$ is in the $x y$ plane (so $S^{z}=0$ ) and let the gyroscope start at $t=0$ on the positive $x$-axis. Solving the Fermi equation is then (effectively) reduced to solving two coupled differential equations (see Ref. 8):

$$
\begin{aligned}
& \frac{d S^{x}}{d t}=\gamma^{2} v^{2} \omega \sin (\omega t)\left(S^{x} \cos (\omega t)+S^{y} \sin (\omega t)\right) \\
& \frac{d S^{y}}{d t}=-\gamma^{2} v^{2} \omega \cos (\omega t)\left(S^{x} \cos (\omega t)+S^{y} \sin (\omega t)\right) .
\end{aligned}
$$

For the initial conditions $\left(S^{x}, S^{y}\right)=(S, 0)$ the solutions can be written as 2

$$
\begin{aligned}
& S^{x}=S[\cos ((\gamma-1) \omega t)+(\gamma-1) \sin (\omega \gamma t) \sin (\omega t)] \\
& S^{y}=S[\sin ((1-\gamma) \omega t)-(\gamma-1) \sin (\omega \gamma t) \cos (\omega t)] .
\end{aligned}
$$

The first term on the right-hand side of Eq. A3a and Eq. (A3b) respectively, corresponds to a rotation about the $z$-axis, but there is also another superimposed rotation with a time dependent amplitude. To find this solution directly from the coupled Fermi equations seems rather difficult, even for this very symmetric and simple scenario.

\section{B Curvature and acceleration}

Suppose that we have an upward accelerating reference frame. A test particle moves with velocity $\mathbf{v}$ along a path, fixed to the reference frame, with the local curvature $R$ and curvature direction $\hat{\mathbf{n}}$. We would like to express the part of the test particle's acceleration that is perpendicular to the particle's momentary direction of motion, relative to an inertial system in which the reference frame is momentarily at rest. For this purpose, we consider how the test particle will deviate from a straight line fixed to the inertial system and directed in the momentary direction of motion of the test particle.

For the small relative velocities between the inertial system and the reference frame that we will consider here, we need not differentiate between the length and time scales of the two systems. Consider a short time step $\delta t$ after the particle has passed the origin. To lowest order with respect to $\delta t$, the perpendicular acceleration relative to the reference frame is given by $v^{2} \hat{\mathbf{n}} / R$. From Fig. 14 we have to lowest nonzero order in $\delta t$

$$
\begin{aligned}
\delta \mathbf{x}_{1} & =\frac{\hat{\mathbf{n}}}{R} \frac{v^{2} \delta t^{2}}{2} \\
\delta \mathbf{x}_{2} & =\mathbf{g}_{\perp} \frac{\delta t^{2}}{2} \\
\delta \mathbf{x}_{3} & =\delta \mathbf{x}_{1}-\delta \mathbf{x}_{2} .
\end{aligned}
$$

Here $\mathbf{g}_{\perp}$ is the acceleration of the inertial system relative to the reference frame (we have $\mathbf{g}_{\perp}=-\left[\mathbf{a}_{\mathrm{ref}}\right]_{\perp}$ ) in the direction perpendicular to the direction of motion. We know that $\delta \mathbf{x}_{3}=\left[\mathbf{a}_{\text {particle }}\right]_{\perp} \frac{\delta t^{2}}{2}$ to lowest order in $\delta t$. If 


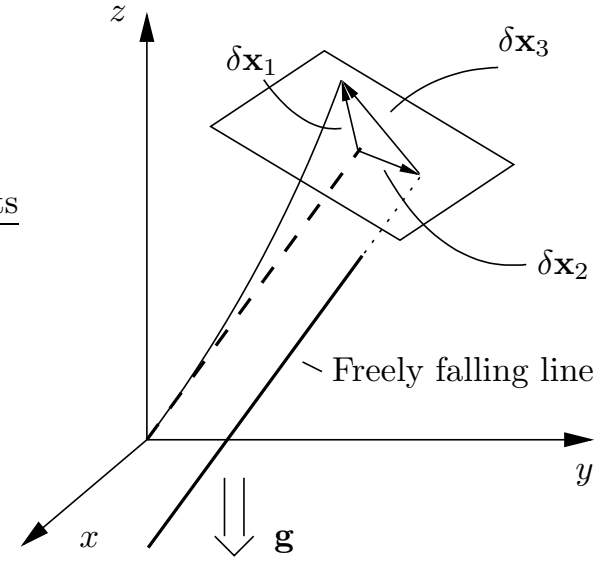

Figure 14: Deviations from a straight line relative to a reference frame that accelerates in the $z$-direction. The plane shown is perpendicular to the momentary direction of motion (along the dashed line), and all the three vectors lie in this plane. The solid curving line is the particle trajectory. The thick line is the line that is fixed to the inertial system in question, and is thus falling relative to the reference frame.

we substitute this expression for $\delta \mathbf{x}_{3}$ into Eq. (B1c) and take the infinitesimal limit, it follows that

$$
\left[\mathbf{a}_{\text {particle }}\right]_{\perp}=\left[\mathbf{a}_{\mathrm{ref}}\right]_{\perp}+v^{2} \frac{\hat{\mathbf{n}}}{R} .
$$

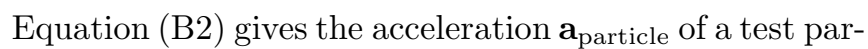
ticle, relative to an inertial system in which the reference frame is momentarily at rest, for given path curvature relative to the reference frame and given acceleration $\mathbf{a}_{\text {ref }}$ of the reference frame.

\section{References}

[1] This angular velocity describes how the gyroscope axis precesses if we imagine that the length contraction along the direction of motion is removed. We will discuss this point further in the text.

[2] C. W. Misner, K. S. Thorne, and J. A. Wheeler, Gravitation (W. H. Freeman, New York, 1973), pp. 175-176.

[3] There is a radius where free photons, that is, photons whose motion is determined by nothing but gravity, can move on circles around a black hole. The circumference of this circle is 1.5 times the circumference of the surface of the black hole (the event horizon).

[4] Reference 2, pp. 1117-1120.

[5] R. D'Inverno, Introducing Einstein's Relativity (Oxford University Press, Oxford, 1998), p. 129.

[6] Two inertial systems $S$ and $S^{\prime}$ are said to be in standard configuration if their spatial axes are aligned,
$S^{\prime}$ moves along the $x$-axis of $S$, and their spatial origins coincide at $t=t^{\prime}=0$. The systems are then related by the standard Lorentz transformation. Two inertial systems $S$ and $S^{\prime}$ are related by a boost assuming that the same rotation of $S$ and $S^{\prime}$ is required to put the two systems into standard configuration.

[7] Consider an infinitesimal boost in a general direction between the $x$ and $y$ axis of $S^{\prime}$. Because relativistic rotation effects vanish at low speeds (according to Eq. (4) $\delta \alpha \simeq v \delta u / 2$ ), the boost is, to first order in the velocity change, equivalent to first boosting the grid in the $x$-direction and then in the $y$-direction. The forward $x$-boost has no impact on the relative rotations. It follows that, to first order in the velocity change, the $y$-part of the boost contributes to the rotation as if there were no simultaneous forward $x$-boost.

[8] R. Jonsson, "A covariant formalism of spin precession with respect to a reference congruence," Class. Quantum Grav. 23, 37-59 (2006).

[9] Note that $\mathbf{g}$ should still be the acceleration of a dropped object relative to the platform. In fact, as observed relative to the train, the acceleration of a dropped object will be greater than $\mathrm{g}$ for nonzero train velocities.

[10] M. A. Abramowicz, "Relativity of inwards and outwards: An example," Month. Not. Roy. Astr. Soc. 256, 710-718 (1992).

[11] Wolfgang Rindler and Volker Perlick, "Rotating coordinates as tools for calculating circular geodesics and gyroscopic precession," Gen. Rel. Grav. 22, 1067-1081 (1990).

[12] Replace $\omega r$ in Eq. (39) of Ref. 11 by $v \sqrt{g_{t t}}$ where $g_{t t}=1-2 M / r$.

[13] S. Weinberg, Gravitation and Cosmology: Principles and Applications of the General Theory of Relativity (John Wiley \& Sons, New York, 1972), pp. 233-238.

[14] R. T. Jantzen, P. Carini and D. Bini, "The many faces of gravitoelectromagnetism," Ann. Phys. (NY) 215, 1-50 (1992). 


\section{Addendum to "Gyroscope preces- sion in special and general relativ- ity from basic principles"}

\section{Rickard M. Jonsson}

Department of Theoretical Physics, Physics and Engineering Physics, Chalmers University of Technology, and Göteborg University, 41296 Gothenburg, Sweden

E-mail: rico@fy.chalmers.se

\begin{abstract}
I extend the reasoning of Rickard Jonsson, Am. Journ. Phys 75 463, (2007) to include also rotating reference frames.
\end{abstract}

\section{Rotating reference frame}

Suppose that the reference frame is accelerating with acceleration $\mathbf{a}_{\text {ref }}$ and rotating (rigidly) with angular velocity $\boldsymbol{\omega}$, relative to an inertial frame $S$ momentarily comoving with a certain point of the reference frame at $t=0$. At this time the gyroscope (grid) is assumed to pass the point in question. The scenario is illustrated in Fig. 1,

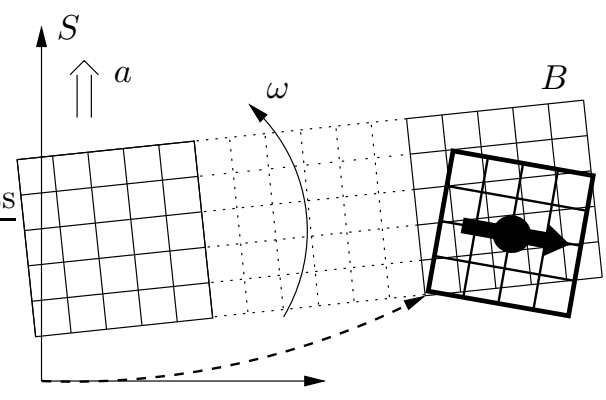

Figure 1: A gyroscope grid (thick lines) moving relative to a rotating and accelerating reference frame (thin lines). Notice that due to the rotation, the part of the reference frame situated at the gyroscope grid at $t=\delta t$ has an extra velocity apart from that coming from the acceleration of the reference frame. This Coriolis related velocity will give an extra contribution to the relative precession, analogous to the already discussed effect of pure accelerations of the reference frame, apart from the obvious non-relativistic effects of the rotation.

Let us denote the part of the reference frame situated at the gyroscope grid at $t=\delta t$ by $B$. Relative to $S, B$ has a small velocity already at $t=0$. At $t=\delta t$, it has to lowest order the velocity $\mathbf{a}_{\text {ref }} \delta t+\boldsymbol{\omega} \times \mathbf{v} \delta t$. To lowest order in $\delta t$ we may consider $B$ to have been at rest in $S$ at $t=0$, but rotated by an angle $\omega \delta t$ around $\hat{\omega}$. It is then accelerated to a velocity $\mathbf{a}_{\text {ref }} \delta t+\boldsymbol{\omega} \times \mathbf{v} \delta t$, hence getting an extra turn from the Thomas precession as seen from a system comoving with the gyroscope grid at $t=\delta t$. With this insight one can modify Eq. 12 of Ref. 1, to include the rotation. We just replace $\mathbf{a}_{\text {ref }}$ by $\mathbf{a}_{\text {ref }}+\boldsymbol{\omega} \times \mathbf{v}$ and add a $-\boldsymbol{\omega}$ term, to account for the mentioned (non-relativistic) rotation, to find

$$
\begin{aligned}
\boldsymbol{\Omega}= & \frac{\gamma}{\gamma+1}\left(\left[\gamma \mathbf{a}_{\mathrm{gyro}}+\left(\mathbf{a}_{\mathrm{ref}}+\boldsymbol{\omega} \times \mathbf{v}\right)\right] \times \mathbf{v}\right) \\
& -\boldsymbol{\omega} .
\end{aligned}
$$

By $\mathbf{a}_{\text {gyro }}$ we here mean the acceleration of the gyroscope relative to the inertial frame $S$. In the non-rotating case we had $\left[\mathbf{a}_{\text {gyro }}\right]_{\perp}=v^{2} \frac{\hat{\mathbf{n}}}{R}+\left[\mathbf{a}_{\text {ref }}\right]_{\perp}$. Now that we have rotation this is modified to $\left[\mathbf{a}_{\text {gyro }}\right]_{\perp}=v^{2} \frac{\hat{\mathbf{n}}}{R}+\left[\mathbf{a}_{\text {ref }}\right]_{\perp}+2 \boldsymbol{\omega} \times \mathbf{v}$. This is a simple Coriolis effect that is easy to derive analogous to the proof in Appendix B of Ref. 1. Using this in Eq. (1) yields

$$
\begin{aligned}
\boldsymbol{\Omega}=\frac{\gamma}{\gamma+1}( & {\left[\gamma v^{2} \frac{\hat{\mathbf{n}}}{R}+\mathbf{a}_{\mathrm{ref}}(\gamma+1)\right.} \\
& +(2 \gamma+1) \boldsymbol{\omega} \times \mathbf{v}] \times \mathbf{v})-\boldsymbol{\omega} .
\end{aligned}
$$

So here is the precession rate of the gyroscope grid (in the stopped sense) relative to an accelerating and rotating reference frame. Comparing Eq. (11) and Eq. (2) with the more formally derived results of Ref. 2 (Eqs. 50 and 51), we find a perfect match.

To apply this formalism to for instance rotating black holes, we must take the spatial geometry into account analogous to the case for static black holes, see Ref. 2.

\section{References}

[1] R. Jonsson, "Gyroscope precession in special and general relativity from basic principles," Am. Journ. Phys. 75, 463 (2007)

[2] R. Jonsson, "A covariant formalism of spin precession with respect to a reference congruence," Class. Quantum Grav. 23, 37-59 (2006). 